Anaesthesist 2019 • 68:827-835 https://doi.org/10.1007/s00101-019-00691-8 Eingegangen: 4. September 2019 Überarbeitet: 6. Oktober 2019 Angenommen: 15. Oktober 2019 Online publiziert: 5. November 2019 () Der/die Autor(en) 2019

\begin{abstract}
Die Gesundheitsausgaben in den meisten Ländern steigen stetig, trotzdem werden fundierte wissenschaftliche Artikel zum Thema Medizinökonomie selten publiziert. Dies gilt auch für das Fachgebiet der Anästhesie sowie deren Subspezialitäten. Insbesondere die Regionalanästhesie hat sich hier während der letzten Jahre kontinuierlich weiterentwickelt, und die Implementierung des Ultraschalls hat zur Effizienzsteigerung auf diesem Gebiet entscheidend beigetragen. Der vorliegende Artikel analysiert anhand von 3 Fallvignetten (anästhesiologische Versorgung einer Unterarmfraktur in suffizienter Regionalanästhesie, Allgemeinnarkose und insuffizienter Regionalanästhesie) die ökonomischen Implikationen der verschiedenen anästhesiologischen Verfahren. Entsprechende Maßnahmen zur erfolgreichen ökonomisch orientierten Implementierung der Regionalanästhesie in den klinischen Alltag werden in diesem Artikel besprochen.
\end{abstract}

\section{Hintergrund}

Die Bedeutung der Regionalanästhesie in der perioperativen Versorgung von Patienten ist unbestritten. Bei fachgerechter Anwendung werden zufriedenstellende Ergebnisse in Bezug auf Erfolgsraten bei sehr geringer Komplikation erzielt. Die Implementierung des

P. Marhofer ${ }^{1}$ M. Mraus ${ }^{2}$ - D. Marhofer ${ }^{1}$

${ }^{1}$ Klinische Abteilung für Anästhesie, allgemeine Intensivmedizin und Schmerztherapie, Medizinische Universität Wien, Wien, Österreich

${ }^{2}$ Institut für Höhere Studien, Wien, Österreich

\title{
Regionalanästhesie im klinischen Alltag: eine ökonomische Analyse anhand eines Versorgungsbeispiels
}

Ultraschalls für periphere und zunehmend auch zentrale Blockadeverfahren stellt in diesem Zusammenhang einen entscheidenden Aspekt zur Qualitätsverbesserung dar. Erst die direkte Visualisierung von neuronalen und umgebenden anatomischen Strukturen, Kanülen sowie der Möglichkeit, Lokalanästhetika exakt $\mathrm{zu}$ platzieren, gestattete, effiziente und sichere regionalanästhesiologische Techniken zu entwickeln $[3,12,16,17,25]$.

Die Effizienz und Sicherheit der Regionalanästhesie in der heutigen Zeit sind also mehr oder weniger unbestritten [15]. Die ökonomischen Aspekte dieser Techniken hingegen sind bisher wenig analysiert. Als eines der Hauptargumente gegen die Durchführung perioperativer regionaler Techniken werden zumeist Zeitaspekte genannt. Regionalanästhesie wird dabei stets, im Verhältnis zum Nutzen, in der klinischen Praxis als zeitaufwendig betrachtet. Chirurgische Alternativen $\mathrm{zu}$ regionalen Verfahren, wie z. B. direkte Infiltrationen in Gelenke, werden in diesem Zusammenhang gerne hervorgebracht. Ohne hier näher in Details zu gehen, sei z. B. im Schulterbereich auf potenzielle schwere systemimmanente Komplikationen dieser chirurgischen Infiltrationen verwiesen [9].

Im Jahre 2009 wurde eine der ersten ökonomischen Untersuchungen zu diesem Thema publiziert [10]. Dabei wurden Schulteroperationen in reiner Regionalanästhesie mit Allgemeinnarkosen in Bezug auf ökonomische Aspekte verglichen. Während die reinen Kosten, welche durch die spezifischen Techniken verursacht wurden, sich zwar signifikant, aber im Kontext der Gesamtkosten von solchen Operationen nur geringfügig voneinander unterschieden (im Durchschnittswert $33 €$ vs. $41 €, p<0,01)$, konnte in der Regionalanästhesiegruppe eine Reduktion von 11 min der Zeit, welche durch die Anästhesie beeinflusst werden kann, erzielt werden. Die Kosten einer Operationssaalminute (die sich aus unterschiedlichsten Parametern zusammensetzt) wurden damals mit $15 €$ berechnet. Somit konnte eine Kostenersparnis durch die fachgerechte Anwendung regionaler Blockaden von durchschnittlich $173 €$ $(11 \min \times 15 €+8 €)$ pro Fall berechnet werden.

Bevor die ökonomischen Aspekte der Regionalanästhesie detaillierter beschrieben werden, müssen einige wichtige Fakten und Zahlen der Gesundheitsfinanzierung angeführt werden.

\section{Gesundheitsausgaben mit Fokus auf den Krankenhausbereich}

Im Jahr 2017 lagen die laufenden Gesundheitsausgaben (inkl. Ausgaben für Langzeitpflege) in Österreich bei $10,4 \%$ des Bruttoinlandprodukts (BIP) $(=38,5 \mathrm{Mrd}$. $€)$ nach System of Health Accounts (SHA). Davon entfielen 74,0\% $(=28,5 \mathrm{Mrd} . €)$ auf die öffentlichen und $26,0 \%(=10,0$ Mrd. $€)$ auf die privaten Ausgaben. Die laufenden Gesundheitsausgaben stellten damit die zweithöchste 
Ausgabenposition nach jener für soziale Sicherung (Ausgaben für Pensionen, Familien, Arbeitslose) des österreichischen Budgets dar. Im Vergleich dazu betrugen im Jahr 2017 die Ausgaben für Bildung 5,5\% des BIP und jene für Rüstung 0,6 \% des BIP [19, 23].

Im OECD-Vergleich liegt Österreich mit einem Anteil der laufenden Gesundheitsausgaben am BIP von 10,4\% deutlich über dem OECD-Durchschnitt von $8,8 \%$. In Europa weisen die Schweiz mit $12,2 \%$ des BIP, Deutschland und Frankreich mit jeweils $11,2 \%$ des BIP sowie die nordischen Länder (Schweden 11,0\%, Dänemark 10,5\% und Norwegen 10,2\%) die höchsten Gesundheitsausgaben und Polen mit $6,3 \%$ des BIP sowie Lettland mit 5,9\% des BIP die geringsten Gesundheitsausgaben auf [19].

Die oben angeführten Zahlen zeigen die Bedeutung des Gesundheitswesens für die nord-west- und mitteleuropäischen Staaten, nicht zuletzt, weil die Bevölkerung eine ununterbrochene einkommensunabhängige medizinische Versorgung auf höchstem Niveau bei ständig steigenden Gesundheitsausgaben erwartet. Insofern ist ein steigendes Kosten- und Effizienzbewusstsein bei allen Stakeholdern im Gesundheitswesen unabdingbar. Dies ist insbesondere auch der Fall aufgrund der anstehenden Herausforderungen, die durch demografische, epidemiologische, technologische und gesellschaftliche Entwicklungen sowie das Baumol-Phänomen [2, 11] hervorgerufen werden.

Ein besonderer Kostentreiber ist dabei ein stark ausgebauter Krankenhaussektor, der im Folgenden am Beispiel Österreichs näher beleuchtet wird [1] .

\section{Stationäre und ambulante Krankenhauskosten und deren Implikationen auf das perioperative Prozessmanagement}

Die öffentlichen Gesundheitsausgaben für die Fondskrankenanstalten (dabei handelt es sich um Akutkrankenanstalten in öffentlicher und privater Trägerschaft, welche im Jahre 2017 87,9\% der stationären Aufenthalte von Patienten stellten und somit die wichtigste Gruppe der österreichischen Krankenhäuser sind), inkl. Krankenhausambulanzen, beliefen sich in Österreich im Jahr 2017 auf 11,48 Mrd.€. Den Großteil der Ausgaben trugen die Sozialversicherungsträger mit einem Ausgabenanteil von 45,5\% (5,22 Mrd.€), gefolgt von den Bundesländern mit 32,6\% (3,74 Mrd.€), dem Bund mit 11,3\% (1,30 Mrd.€) und den Gemeinden mit 10,6\% (1,22 Mrd.€) [23].

Ein Aufenthalt in einer Fondskrankenanstalt kostete im Jahr 2017 durchschnittlich $4952 €$ (exkl. kalkulatorische Anlagekapitalkosten) [4]. Die Versorgung in einer Krankenhausambulanz kostete im Jahr 2017 im Durchschnitt $261 € /$ Patient (in Wien 297€) [4] und ist somit deutlich günstiger als die stationäre Versorgung. Wann immer also eine ambulante Versorgung möglich ist, sollte sie angeboten und realisiert werden. Dies muss natürlich ohne Leistungseinschränkung (hier sei auf die postoperative Schmerztherapie im Besonderen hingewiesen) geschehen.

Prinzipiell kann das österreichische Gesundheitswesen als Beispiel eines sehr krankenhauslastigen Versorgungssystems herangezogen werden [1], was bedeutet, dass es stationäre Aufenthalte favorisiert. Das spiegelt sich z. B. in einer sehr hohen Akutbettendichte und einem sehr geringen Anteil an ambulanter Chirurgie wider. Demgegenüber stehen Publikationen, welche die ökonomischen Vorteile der ambulanten Chirurgie beschreiben [22]. Ein verbessertes perioperatives Prozessmanagement und der Einsatz der Regionalanästhesie könnten sich dabei positiv auf den Anteil an ambulant durchgeführten Operationen auswirken. Aufgrund dessen wird im Folgenden die Bedeutung des perioperativen Prozessmanagements kurz erläutert.

Im perioperativen Prozessmanagement stellen Zeit und Personal zwei der wichtigsten Kostenfaktoren dar. Schon die oben erwähnte 2009 publizierte ökonomische Analyse zeigte die signifikante Zeitersparnis einer weitgehend prozessoptimierten Regionalanästhesie [10]. Der Vorteil der Regionalanästhesie ist die zeitliche Gestaltungsmöglichkeit. Regionalanästhesie per se ist nicht schneller als Allgemeinanästhesie, allerdings können Prozesse im Bereich der Regionalan- ästhesie parallel ablaufen. So kann das anästhesiologische Management genau geplant werden und findet im Optimalfall bereits während der Beendigung der vorhergehenden Operation statt (oder während der Operationssaal gereinigt wird und die hygienebedingten Trocknungszeiten abgewartet werden müssen). Die Zeit der Blockadeanlage kann somit von der Operationszeit abgezogen werden, was mit Zeit- und Personalkostenersparnis gleichzusetzen ist. Ziel sollte immer sein, Pausen zwischen zwei Operationen möglichst kurz zu halten, da ein nichtgenutzter Operationssaal kostenintensiv ist. Weiters muss die Durchführung geplanter Operationen gewährleistet sein, da Verschiebungen in der Regel einen finanziellen Mehraufwand für die Krankenhäuser bedeuten. Spätestens hier ist klar, dass Kostenfaktoren im perioperativen Bereich nicht einzeln betrachtet und analysiert werden können. Optimales Prozessmanagement eines Operationsprogrammes ist nur realisierbar, wenn keine unvorhergesehenen Ereignisse auftreten (also keine Zeitverzögerungen im anästhesiologischen Bereich z.B. durch nichtfunktionierende regionalanästhesiologische Blockaden oder im chirurgischen Bereich z.B. durch Überschreitungen der kalkulierten Operationszeiten). Dies setzt Folgendes voraus: einerseits eine entsprechende Ausbildung des gesamten involvierten Personals und andererseits ein funktionierendes interdisziplinäres Zusammenspiel. In diesem Zusammenhang sei auch auf die wichtige Rolle des Pflegepersonals, administrativen Personals, aber auch des Krankentransportdienstes und des Reinigungsdienstes hingewiesen.

Die oben zitierte Publikation von Gonano et al. [10] wurde schon anhand genauer Datensätze erstellt. Allerdings fehlte hier die ökonomisch-wissenschaftliche Sichtweise. Der vorliegende Untersuchung basiert auf Datensätzen, welche zur Erstellung einer gesperrten Masterthese zur Erlangung des akademischen Grads Master of Business Administration (Universitätslehrgang Health Care Management der Medizinischen Universität Wien) erhoben wurden und verfolgt zwei Ziele: 1) Durchführung einer öko- 
nomischen Analyse des perioperativen Prozessmanagements anhand verschiedener Narkoseverfahren mittels dreier Fallvignetten (anästhesiologische Versorgung einer Unterarmfraktur in reiner Regionalanästhesie vs. reine Allgemeinnarkose vs. insuffiziente Regionalanästhesie) und 2) Durchführung einer SWOT-Analyse der ultraschallgezielten Regionalanästhesie (englisches Akronym für $S$,strengths“, $W$,weaknesses“, $O$ „opportunities“ und $T$,threats").

Anhand der beiden Ziele wird regionalanästhesiologische mit ökonomischer Expertise verknüpft, und mögliche Implikationen auf die tägliche klinische Arbeit werden besprochen.

\section{Material und Methodik}

Ökonomische Aspekte des perioperativen Prozessmanagements aus Sicht der ultraschallgezielten Regionalanästhesie, dargestellt mittels Fallvignetten

Im Folgenden werden die ökonomischen Aspekte des perioperativen Prozessmanagements anhand verschiedener Narkoseverfahren beleuchtet. Hierzu werden drei Fallvignetten beschrieben.

Fallvignetten als methodisches Instrument beschreiben imaginäre Situationen, wodurch Prozesse nachvollzogen werden können. Fallvignetten bestehen aus bestimmten Bausteinen und stellen verschiedene Varianten dar. Die spezifischen Erkenntnisse der Fallvignetten können dann auf andere Fälle übertragen und generalisiert werden. Fallvignetten eignen sich als empirisches Verfahren, wenn randomisierte Studien sehr aufwändig und große Fallzahlen erforderlich wären und die Ergebnisse als ähnlich zu erwarten/ bewerten sind $[7,8,20]$.
Die beschriebenen Fallvignetten beinhalten folgende Bausteine: 1) Personalkosten, 2) Kosten der Ge- und Verbrauchsgüter, 3) Kosten der Anlagegüter und 4) anästhesierelevante Gemeinkosten. Diese Kostenkalkulationen beruhen auf Daten und Berechnungen des Wiener Allgemeinen Krankenhauses (AKH). Das Wiener AKH ist eines der größten Krankenhäuser Europas und verfügt über 2098 Betten und verzeichnete im Jahr 2017120.186 stationäre Aufnahmen und 1.099.110 ambulante Frequenzen (davon 536.069 ambulante Erstbesuche und 563.041 ambulante Folgebesuche). Dies wird mit ca. 1500 ärztlichen und 7300 nichtärztlichen Mitarbeitern geleistet. Die Kostenstruktur des Wiener AKH kann sicher mit anderen Häusern dieser Größenordnung und zentralem Versorgungsauftrag verglichen werden.

\section{Berechnung der Personalkosten}

Hier macht es einen Unterschied, ob ein Krankenhaus einen reinen Versorgungsauftrag oder darüber hinaus auch einen

Hier steht eine Anzeige. 
Lehr- und Forschungsauftrag innehat. Ein solcher Mehraufwand (klinischer Mehraufwand) wird in der Finanzierung berücksichtigt und spiegelt sich z. B. in den Personalkosten des Wiener Krankenanstaltenverbundes (KAV) vs. des AKH als Universitätsspital wider. Somit wird ärztliches Personal im KAV (ohne Lehr- und Forschungsauftrag) mit 1,56€/min (Pflegepersonal mit $0,72 € / \mathrm{min}$ ), im AKH (mit Lehr- und Forschungsauftrag) mit $1,63 € / \mathrm{min}$ und $0,74 € /$ min berechnet.

\section{Berechnung der Ge- und Verbrauchsgüter}

Diese werden nach Preislisten des Einkaufs der jeweiligen Wirtschaftsabteilungen berechnet. Medikamente werden nach Preislisten der Anstaltsapotheken berechnet. Diese Preislisten sind für Führungskräfte frei zugänglich.

\section{Berechnung der Anlagegüter}

Eine medizinische Anlage hat zum Zeitpunkt der Anschaffung einen bestimmten Wert, der über eine bestimmte Anzahl an Jahren abgeschrieben wird. Die Berechnung der Anlagekosten pro Jahr richtet sich nach dem Anschaffungswert und der kalkulierten Nutzungsdauer. Hinzu kommen eine Servicepauschale und die kalkulierten Zinsen. Dies alles setzt sich wie folgt zusammen:

$$
\begin{aligned}
& \text { Anschaffungswert/Nutzungsdauer in } \\
& \text { Jahren = jährliche Abschreibungsquote } \\
& \text { (Absetzung für Abnützung = „Afa“) } \\
& \text { Jährliche Afa-Quote + 13,35\% vom } \\
& \text { Anschaffungswert für Service } \\
& \text { + 0,46\% vom Anschaffungswert } \\
& \text { für kalkulierte Zinsen } \\
& \text { = jährliche Anlagekosten }
\end{aligned}
$$

Um die exakte Berechnung pro Untersuchung zu erhalten, wird folgende Formel herangezogen:

$$
\begin{aligned}
& \text { Kosten pro Untersuchung }= \\
& \text { jährliche Anlagekosten } \\
& \frac{78.000 \text { min }(=5 d \times 5 h \times 52 \text { Wochen })}{\times \text { Untersuchungsdauer in } \mathrm{min}}
\end{aligned}
$$

Anaesthesist 2019-68:827-835 https://doi.org/10.1007/s00101-019-00691-8

(c) Der/die Autor(en) 2019

\section{P. Marhofer · M. Kraus · D. Marhofer \\ Regionalanästhesie im klinischen Alltag: eine ökonomische Analyse anhand eines Versorgungsbeispiels}

\section{Zusammenfassung}

Hintergrund. Ein wichtiger Aspekt jedes medizinischen Verfahrens ist dessen ökonomische Wirkungsweise. Mithilfe von Fallvignetten werden die ökonomischen Implikationen verschiedener anästhesiologischer Verfahren analysiert. Methoden. Basierend auf 3 Fallvignetten (anästhesiologische Versorgung einer Unterarmfraktur in suffizienter Regionalanästhesie, Allgemeinnarkose und insuffizienter Regionalanästhesie) werden die anästhesiologisch relevanten Kostenfaktoren (Personalkosten, Kosten für Ge- und Verbrauchsgüter, Kosten für Anlagegüter, anästhesierelevante Gemeinkosten) errechnet.

Ergebnisse. Die ausschließliche Regionalanästhesie für den genannten chirurgischen Eingriff ist mit $324,26 €$ am kostengünstigsten, gefolgt von der Allgemeinnarkose mit $399,18 €(+23 \%$ im Vergleich zur Regional- anästhesie). Am kostenungünstigsten ist die insuffiziente Regionalanästhesie, die eine zusätzliche Allgemeinnarkose erforderlich macht $(482,55 €,+49 \%)$, im Vergleich zur suffizienten Regionalanästhesie.

Schlussfolgerungen. Auch unter Berücksichtigung der Tatsache, dass die Kostenanalyse an nur einer Institution durchgeführt wurde (Allgemeines Krankenhaus der Stadt Wien, Universitätskliniken), erscheint die suffiziente Regionalanästhesie im Vergleich zu anderen anästhesiologischen Verfahren als kostengünstig. Hauptkostentreiber in Bezug auf das anästhesiologische Management einer Unterarmfraktur sind Personalkosten.

Schlüsselwörter

Leitungsanästhesie · Allgemeinanästhesie . Kosten und Kostenanalyse - Medizinische Ökonomie · Gesundheitsökonomie

\section{Regional anesthesia in daily clinical practice: an economic analysis based on case vignettes}

Abstract

Background. The economic effect is a crucial aspect of every medical procedure. This article analyzes the economic implications of various methods in anesthesia based on three case vignettes.

Methods. The management of anesthesia of a forearm fracture with sufficient brachial plexus blockade, general anesthesia and insufficient brachial plexus blockade with subsequent general anesthesia was analyzed with respect to the relevant cost factors (personnel costs, durables, consumables, fixed assets costs, anesthesia-related overhead costs).

Results. Sufficient regional anesthesia was the least expensive method for a forearm fracture with $324.26 €$, followed by general anesthesia with $399.18 €(+23 \%$ compared with regional anesthesia). Insufficient regional anesthesia was most the expensive method, which necessitated an additional general anesthesia ( $482.55 €,+49 \%$ compared with sufficient regional anesthesia).

Conclusion. Even considering that this cost analysis was calculated based on data from only one medical institution (General Hospital of Vienna, Medical University of Vienna), regional anesthesia appeared to be cost efficient compared with other anesthesia procedures. Main cost drivers in this example were personnel costs.

\section{Keywords}

Anesthesia, conduction - Anesthesia, general . Costs and costs analysis - Economics, medical . Health care economics and organizations
Großgeräte haben in der Regel eine Nutzungsdauer von 10 Jahren. Die Nutzungsdauern kleinerer Geräte sind einer Liste der Abteilung für Kostenrechnung zu entnehmen. Für das anästhesierelevante Ultraschallgerät wird eine Nutzungsdauer von 7 Jahren unterstellt. Somit würde unter Heranziehung der obe- ren Formeln eine 10-minütige Nutzung für die Anlage einer regionalanästhesiologischen Blockade mit einem 40.000€ teuren Ultraschallgerät mit $1,44 €$ berechnet werden. 


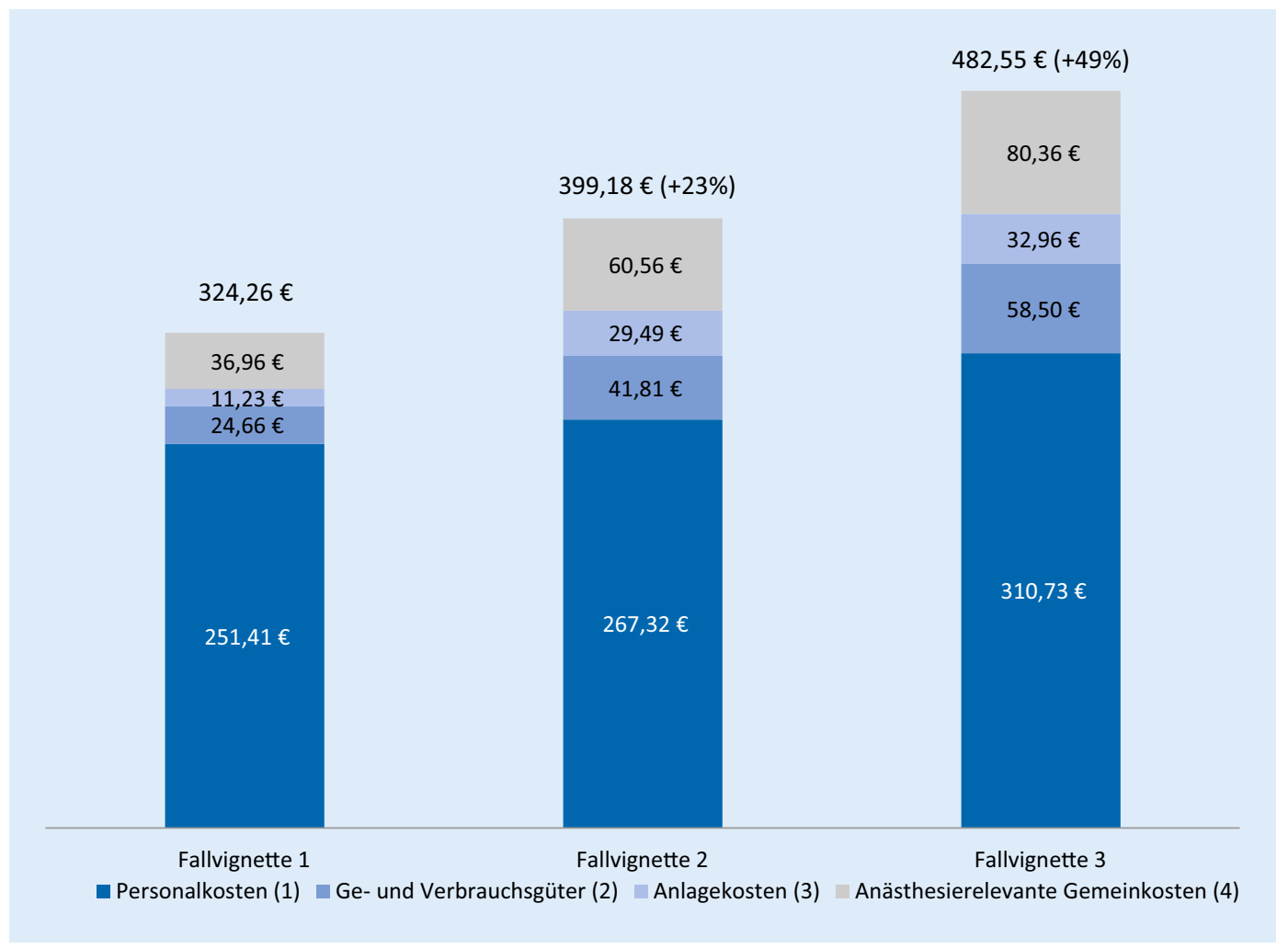

Abb. 1 ॥ Zusammensetzung der verschiedenen Kosten der drei Fallvignetten. (1) Zusammensetzung Personalkosten Fallvignette 1:1 × Anästhesistln (125 min), 0,5 ×DGKP (diplomierte Gesundheits- und Krankenpflegeperson), Anästhesie-OP (112 min), 0,3 × DGKP, Anästhesie-Aufwachraum (28 min).Zusammensetzung Personalkosten Fallvignette 2: $1 \times$ Anästhesistln (127 min), 0,5 × DGKP, Anästhesie-OP (127 min), 0,3 ×DGKP, Anästhesie-Aufwachraum (60 min). Zusammensetzung Personalkosten Fallvignette 3: $1 \times$ Anästhesistln (148 min), 0,5 ×DGKP, Anästhesie-OP (135 min), 0,3 ×DGKP, Anästhesie-Aufwachraum (88 min). (2) Zusammensetzung Ge- und Verbrauchsgüter Fallvignette 1:1×Anästhesie-Aufklärungsbogen, $2 \times$ unsterile Handschuhe (Stück), $3 \times$ EKG-Elektrode, $1 \times$ Isozidalkohollösung, $1 \times$ Stauband, $1 \times$ Venenverweilkanüle, $1 \times$ Venflon-Fixierpflaster, $1 \times \mathrm{NaCl}, 0,9 \%$ ig, $1 \times$ Dreiwegehahn, $1 \times$ Dreiwegehahn, Verlängerung, $10 \mathrm{~cm}, 3 \times$ Kombistopfen, $1 \times$ Elomel-Isoton-Infusionslösung, $1 \times$ Aufziehnadel, $1 \times$ sterileHandschuhe $($ Paar), $1 \times$ Plexusnadel, $50 \mathrm{~mm}, 1 \times$ Ultraschallabdeckfolie, $1 \times$ Ultraschallgel, $1 \times$ Ropinaest, $7,5 \mathrm{mg} / \mathrm{ml}, 10 \mathrm{ml}, 1 \times$ Spritze, $2 \mathrm{ml}, 1 \times$ Spritze, $10 \mathrm{ml}, 5 \times$ Tupfer, $1 \times$ Flächendesinfektionsmittel, $5 \times$ Flächendesinfektionsmittel, Tücher, $4 \times$ Einwegputztuch, $1 \times$ Entsorgungsbox, $2 \times$ Klemme, $1 \times$ Müllsack, orange. Zusammensetzung Ge- und Verbrauchsgüter Fallvignette 2: $1 \times$ Anästhesie-Aufklärungsbogen, $8 \times$ unsterile Handschuhe (Stück), $3 \times$ EKG-Elektrode, $1 \times$ StaubandfürBlutabnahme, $1 \times$ Isozidakohollösung, $1 \times$ Venenverweilkanüle, $1 \times$ Venflon-Fixierpflaster, $1 \times \mathrm{NaCl}, 0,9 \%$ ig, $1 \times$ Elomel-Isoton-Infusionslösung, $1 \times$ Dreiwegehahn, $1 \times$ Dreiwegehahn, Verlängerung, $10 \mathrm{~cm}, 9 \times$ Kombistopfen, $5 \times$ Aufziehnadel, $1 \times$ Atemkalk, $1 \times$ Narkoseschlauchset, $1 \times$ Beatmungsbeutel, $1 \times$ Beatmungsmaske, $0,3 \times$ Sauerstoff, Med-Air, $1 \times$ Filter mit Monitoranschluss, $1 \times$ Spritze, $2 \mathrm{ml}, 1 \times$ Spritze, $5 \mathrm{ml}, 1 \times$ Spritze, $10 \mathrm{ml}, 1 \times$ Spritze, $20 \mathrm{ml}, 1 \times$ Dormicum, $5 \mathrm{mg} / 5 \mathrm{ml}, 1 \times$ Fentanyl, $0,5 \mathrm{mg} / 10 \mathrm{ml}, 1 \times$ Propofol, $1 \%$ ig, $20 \mathrm{ml}, 1 \times$ Rocuronium, $50 \mathrm{mg}, 0,125 \times$ Sevofluran, $1 \times$ Effortil, $10 \mathrm{mg} / \mathrm{ml}, 1 \times$ Endotrachealtubus, 7,5,1 $1 \times$ Silikonspray, $1 \times$ Mandrineinführhilfe, $1 \times$ Cuffdruckmesser, $1 \times$ Verbindungsschlauch zum Cuffdruck, $1 \times$ Tubusfixierung, $1 \times$ UlmerFixator, $1 \times$ Katheterabsaugschlauch, $2 \times$ Augenschutzpflaster, $2 \times$ Pumpenleitung $1 \times$ Verbindungsschlauch, $1 \times$ Flächendesinfektionsmittel, $5 \times$ Flächendesinfektionsmittel, Tücher, $4 \times$ Einwegputztuch, $1 \times$ Entsorgungsbox, $2 \times$ Klemme, $1 \times$ Müllsack, orange, $5 \times$ Tupfer. (3) Zusammensetzung Ge- und Verbrauchsgüter Fallvignette 3: $1 \times$ Anästhesie-Aufklärungsbogen, $10 \times$ unsterile Handschuhe (Stück), $3 \times$ EKG-Elektrode, $1 \times$ Isozidalkohollösung, $1 \times$ Stauband für Blutabnahme, $1 \times$ Venenverweilkanüle, $1 \times$ Venflon-Fixierpflaster, $1 \times \mathrm{NaCl}, 0,9 \%$ ig, $1 \times$ Dreiwegehahn, $1 \times$ Dreiwegehahn, Verlängerung, $10 \mathrm{~cm}, 9 \times$ Kombistopfen, $1 \times$ Elomel-Isoton-Infusionslösung, $6 \times$ Aufziehnadeln, $1 \times$ sterile Handschuhe (Paar), $1 \times$ Plexusnadel, $50 \mathrm{~mm}, 1 \times$ Ultraschallabdeckfolie, $1 \times$ Ultraschallgel, $1 \times$ Ropinaest, $7,5 \mathrm{mg} / \mathrm{ml}, 10 \mathrm{ml}$, $1 \times$ Spritze, $2 \mathrm{ml}, 1 \times$ Spritze, $5 \mathrm{ml}, 3 \times$ Spritze, $10 \mathrm{ml}, 1 \times$ Spritze, $20 \mathrm{ml}, 1 \times$ Atemkalk, $1 \times$ Narkoseschlauchset, $1 \times$ Beatmungsbeutel, $1 \times$ Beatmungsmaske, $0,3 \times$ Sauerstoff, Med-Air, $1 \times$ Filter mit Monitoranschluss, $1 \times$ Dormicum, $5 \mathrm{mg} / \mathrm{ml}, 1 \times$ Fentanyl, $0,5 \mathrm{mg} / 10 \mathrm{ml}, 1 \times$ Propofol, $1 \% \mathrm{ig}, 20 \mathrm{ml}, 1 \times$ Rocuronium, $50 \mathrm{mg}, 1 \times$ Effortil, $10 \mathrm{mg}, 0,125 \times$ Sevofluran, $1 \times$ Endotrachealtubus, 7,5,1 $1 \times$ Silikonspray, $1 \times$ Mandrineinführhilfe, $1 \times$ Cuffdruckmesser, $1 \times$ Verbindungsschlauch zum Cuffdruck, $1 \times$ Tubusfixierung, $1 \times$ Ulmer Fixator, $1 \times$ Katheterabsaugschlauch, $2 \times$ Augenschutzpflaster, $2 \times$ Pumpenleitung, $1 \times$ Verbindungsschlauch, $1 \times$ Flächendesinfektionsmittel, $5 \times$ Flächendesinfektionsmitteltücher, $4 \times$ Einwegputztuch, $1 \times$ Entsorgungsbox, $2 \times$ Klemme, $1 \times$ Müllsack, orange, $5 \times$ Tupfer. (4) Anlagekosten Fallvignette 1: Narkosewagen, OP-Hocker, Überwachungsmonitor, Ultraschallgerät. Anlagekosten Fallvignette 2: Narkosegerät, Narkosewagen, OP-Hocker, Überwachungsmonitor. Anlagekosten Fallvignette 3: Ultraschallgerät, Narkosegerät, Narkosewagen, OP-Hocker, Überwachungsmonitor. (5) Anästhesierelevante Gemeinkosten Fallvignette 1: OP-Saal (112 min), Aufwachraum (28 min). Anästhesierelevante Gemeinkosten Fallvignette 2: OP (127 min), Aufwachraum (60 min). Anästhesierelevante Gemeinkosten Fallvignette 3: OP-Saal (135 min), Aufwachraum (88 min) 
Tab. 1 Zeitanalyse der drei Fallvignetten (Fallvignette 1: anästhesiologische Versorgung einer Unterarmfraktur in suffizienter Regionalanästhesie, Fallvignette 2: anästhesiologische Versorgung einer Unterarmfraktur in Allgemeinnarkose, Fallvignette 3: anästhesiologische Versorgung einer Unterarmfraktur in insuffizienter Regionalanästhesie, gefolgt von einer Allgemeinnarkose). Die kursivierten Zahlenwerte beschreiben die Zeiten im Operationssaal

\begin{tabular}{|c|c|c|c|}
\hline Prozesse & $\begin{array}{l}\text { Fallvignette } 1 \\
\text { [min] }\end{array}$ & $\begin{array}{l}\text { Fallvignette } 2 \\
\text { [min] }\end{array}$ & $\begin{array}{l}\text { Fallvignette } 3 \\
\text { [min] }\end{array}$ \\
\hline $\begin{array}{l}\text { Vorbereitungszeit inkl. Venenverweil- } \\
\text { kanüle }\end{array}$ & 6 & - & 6 \\
\hline $\begin{array}{l}\text { Ultraschalluntersuchung und Setzen der } \\
\text { Plexusanästhesie }\end{array}$ & 7 & - & 7 \\
\hline Anschlagzeit des Lokalanästhetikums & 15 & - & 15 \\
\hline Einschleuszeit & 7 & 7 & 7 \\
\hline Anlegen des Monitorings im OP & 4 & 4 & 4 \\
\hline $\begin{array}{l}\text { Abwarten auf evtl. verlängerte Anschlag- } \\
\text { zeit des Lokalanästhetikums }\end{array}$ & - & - & 10 \\
\hline Setzen einer Venenverweilkanüle & - & 2 & - \\
\hline Einleitung der Allgemeinnarkose & - & 5 & 5 \\
\hline Operationszeit & 90 & 90 & 90 \\
\hline Ausleitung der Allgemeinnarkose & - & 8 & 8 \\
\hline Abnehmen des Monitorings & 4 & 4 & 4 \\
\hline Ausschleuszeit & 7 & 7 & 7 \\
\hline Aufwachraum (postoperative Observanz) & - & 60 & 60 \\
\hline Summe (min) & 140 & 187 & 223 \\
\hline
\end{tabular}

\section{Berechnung der anästhesie- relevanten Gemeinkosten}

Diese setzen sich aus Raumkosten, Licht, Strom, von der Allgemeinheit verwendete Geräte (z.B. Telefon oder PagerAnlagen) etc. zusammen. Die Raumkosten werden pro $\mathrm{m}^{2}$ angegeben und unterscheiden sich ebenfalls zwischen den verschiedenen Institutionen (KAV:

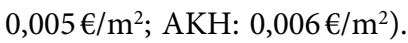

Die Gemeinkosten setzen sich wie folgt zusammen:

$$
\begin{aligned}
& \text { Raumkosten }+25,46 \% \text { Verwaltungs- } \\
& \text { gemeinkosten }+13,35 \% \text { Beschaffungs- } \\
& \text { und Lagerungskosten }
\end{aligned}
$$

\section{Fallvignetten}

Im Folgenden werden auf Basis von drei Fallvignetten verschiedene anästhesiologische Versorgungsmöglichkeiten der operativen Therapie einer Unterarmfraktur beschrieben und die anästhesierelevanten Kosten (• Abb. 1) dargestellt. Diese Fallvignetten wurden im Rahmen einer Masterthese zur Erlangung des „Master of Business Administration“ sie dauert $7 \mathrm{~min}$, und dem Anästhesisten wird von einer Pflegefachkraft assistiert. Fünfzehn Minuten nach erfolgreicher Anlage der axillären Plexusblockade (dies wird mittels Pinprick-Test für die Sensorik und motorischen Tests für den Verlust der Motorik evaluiert) wird der Patient in den Operationssaal eingeschleust. Postoperativ kann der Patient unter Umgehung des Aufwachraums sofort auf die Normalstation verlegt werden.

\section{Fallvignette 2: anästhesiologisches Management einer Unterarmfrak- tur mittels Allgemeinnarkose}

Der 50-jährige Patient, welcher beim Fahrradfahren zu Sturz gekommen und sich den Unterarm gebrochen hat, lehnt aus psychischen Gründen jedwede Regionalanästhesie ab. Deshalb wird nach Abwarten der Nüchternheit eine Allgemeinnarkose durchgeführt. Für das Einleiten der Allgemeinnarkose werden 5 min, für das Ausleiten der Allgemeinnarkose $8 \mathrm{~min}$ berechnet. Postoperativ wird der Patient $60 \mathrm{~min}$ im Aufwachraum betreut, um anschließend auf die Normalstation transferiert zu werden. gesperrt, und die Daten können somit für die vorliegende Originalarbeit verwendet werden.

\section{Fallvignette 1: anästhesiologisches Management einer Unterarmfrak- tur mittels ultraschallgezielter Regionalanästhesie}

Ein 50-jähriger Patient ist beim Fahrradfahren zu Sturz gekommen und weist neben diversen Abschürfungen auch eine sehr schmerzhafte Schwellung des rechten Unterarms auf. Er kommt selbstständig in die Unfallambulanz des AKH, und anhand des Röntgens wird eine Unterarmfraktur diagnostiziert. Nachdem der Patient schmerzgeplagt ist, wird eine Anamnese erhoben und nach Legen einer Venenverweilkanüle und Anlage des Standardmonitorings zügig eine ultraschallgezielte axilläre Plexusanästhesie durchgeführt. Dies alles wird außerhalb des Operationssaals (wo eine vorhergehende Operation gerade beendet wird) im Aufwachraum (in einem eigens für die Anlage von Regionalanästhesien abgetrennten Bereich) durchgeführt. Der Prozess der Anlage der Regionalanästhe-

\section{Fallvignette 3: anästhesiologisches Management einer Unter- armfraktur nach insuffizienter Regionalanästhesie mittels Allgemeinnarkose}

Derselbe Patient, wie oben beschrieben, erhält zuerst eine axilläre Plexusanästhesie, die aber auch nach 15 min zusätzlicher Wartezeit nicht suffizient wirkt. Deshalb muss eine Allgemeinnarkose durchgeführt werden.

\section{SWOT-Analyse} Fallvignetten wird eine SWOT-Analyse erstellt.

\section{Ergebnisse}

Die exakten Zeiten und Personalberechnungen sowie die Zusammensetzung der Personalkosten, Ge- und Verbrauchsgüter, Anlagegüter und anästhesierelevante Gemeinkosten der beschriebenen Fallvignetten sind den • Tab. 1, 2 und der
Basierend auf den oben beschriebenen 
Tab. 2 Analyse der anästhesiebezogenen Personalkosten der drei Fallvignetten (Fallvignette 1: anästhesiologische Versorgung einer Unterarmfraktur in suffizienter Regionalanästhesie, Fallvignette 2: anästhesiologische Versorgung einer Unterarmfraktur in Allgemeinnarkose, Fallvignette 3: anästhesiologische Versorgung einer Unterarmfraktur in insuffizienter Regionalanästhesie, gefolgt von einer Allgemeinnarkose)

\begin{tabular}{llll}
\hline Personal & Fallvignette $\mathbf{1}$ & Fallvignette $\mathbf{2}$ & Fallvignette $\mathbf{3}$ \\
\hline Anästhesist (Anzahl 1) & $125 \min \times 1,63 € \times 1=203,75 €$ & $127 \min \times 1,63 \times 1=207,01 €$ & $148 \min \times 1,63 € \times 1=241,24 €$ \\
DGKP, Anästhesie OP (Anzahl 0,5) & $112 \min \times 0,74 € \times 0,5=41,44 €$ & $127 \min \times 0,74 € \times 0,5=46,99 €$ & $135 \min \times 0,74 € \times 0,5=49,95 €$ \\
\hline DGKP, Anästhesie Aufwachraum (Anzahl 0,3) & $28 \min \times 0,74 € \times 0,3=6,22 €$ & $60 \min \times 0,74 € \times 0,3=13,32 €$ & $88 \min \times 0,74 € \times 0,3=19,54 €$ \\
Gesamtkosten & $251,41 €$ & $267,32 €$ & $310,73 €$
\end{tabular}

DGKP diplomierte Gesundheits- und Krankenpflegeperson

- Abb. 1 zu entnehmen. Es kann eine beinahe lineare Steigerung der anästhesierelevanten Kosten von ausschließlicher Regionalanästhesie zu Allgemeinnarkose $(+23 \%)$ und weiter zur insuffizienten Regionalanästhesie mit danach erforderlichen Allgemeinnarkose (+49\%) festgestellt werden.

Die Kostenersparnis einer suffizienten Regionalanästhesie zieht sich hier durch alle Teilbereiche (Personalkosten, Kosten für Ge- und Verbrauchsgüter, Kosten für Anlagegüter, anästhesierelevante Gemeinkosten), wobei sich die Personalkosten einer insuffizienten Regionalanästhesie im Vergleich zu einer suffizienten Regionalanästhesie um $24 \%$ erhöhen, was in Anbetracht der relativen Kostenstruktur (Personalkosten machen im Falle einer suffizienten Regionalanästhesie $76 \%$ der Gesamtkosten aus) der größte Kostentreiber ist.

Die anhand der oben beschriebenen Ergebnisse erstellte SWOT-Analyse ist in - Abb. 2 dargestellt.

\section{Diskussion}

Die vorliegende Untersuchung hatte die Gegenüberstellung verschiedener anästhesiologischer Methoden bei einer klar definierten chirurgischen Intervention zum Ziel. Die rein anästhesiebezogenen Kosten einer Allgemeinnarkose für eine Unterarmfraktur sind dabei $23 \%$ höher im Vergleich zu einer suffizienten Regionalanästhesie $(=+74,92 €)$. Am ökonomisch ungünstigsten ist dabei eine insuffiziente Regionalanästhesie, die durch eine Allgemeinnarkose ergänzt werden muss $(+49 \%=+158,29 €$ für die beschriebene chirurgische Intervention).

Im AKH Wien werden jährlich ca. 55.000 Operationen mit einem Narkoseverfahren durchgeführt. Abhängig von der theoretischen Anzahl an operativen Eingriffen in ausschließlicher Regionalanästhesie kann hier von einem nichtunbeträchtlichen Einsparungspotenzial ausgegangen werden. Voraussetzung sind aber eben strukturelle Adaptationen, um Regionalanästhesie suffizient durchführen zu können. Grundlage eines entsprechenden Prozessmanagements sind (in Analogie zu den oben beschriebenen und analysierten Fallvignetten) exakte Analysen aller infrage kommenden Eingriffe.

\section{SWOT-Analyse der ultraschall- gezielten Regionalanästhesie}

Wie die ökonomische Analyse gezeigt hat, kann State-of-the-Art-Regionalanästhesie zu einer Kostenreduktion im Krankenhausbereich beitragen, allerdings ist hier ein Umdenken von allen, an perioperativen Prozessen Beteiligten notwendig. Um ein Umdenken einzuleiten und die Chance zur praktischen Implementierung von neuen Verfahren zu nützen, bedient sich das „change management" gerne strategischer Werkzeuge. Ein solches ist die SWOT-Analyse. Eine SWOT-Analyse zielt auf eine möglichst neutrale Darstellung der Stärken, Schwächen, Chancen und Risiken neuer zu implementierender Verfahren ab [24].

Im vorliegenden Überblicksartikel wird die ultraschallgezielte Regionalanästhesie mit einem speziellen Fokus auf deren ökonomische Aspekte unter Zuhilfenahme einer SWOT-Analyse bewertet (• Abb. 2).

Eine Stärke der Regionalanästhesie ist deren Kosteneffizienz in Vergleich zur Allgemeinnarkose. Diese setzt sich aus der zeitlichen Optimierung, der Einsparung von Ge- und Verbrauchsgütern und des evtl. „bypass“ des Aufwach- raums (bei reiner peripherer Regionalanästhesie) zusammen. Durch weitestgehend optimierte Planungsabläufe (z. B. Durchführung der regionalen Blockade, während der Operationssaal gereinigt wird) können zusätzliche Personal- und Raumkosten eingespart werden. Ebenso fallen die Narkoseausleitzeiten weg. Eine weitere Stärke der Regionalanästhesie ist die wenig invasive, aber sehr sichere und effiziente Versorgung multimorbider Patienten. Auch wenn dies noch nicht durch robuste Studien untermauert ist, so liegt es doch auf der Hand, dass die Vermeidung hämodynamisch wirksamer Medikamente oder künstlicher Beatmung für ein multimorbides Patientenkollektiv belastender ist als eine periphere Nervenblockade. Der Ischiadikusblock für Zehen- bzw. Vorfußamputationen bei schweren diabetischen Spätschäden sei hier als nur ein Beispiel genannt [18].

Nachdem kein medizinisches Verfahren ausschließlich Vorteile aufweist, müssen natürlich auch die Schwächen bzw. nachteiligen Aspekte der Regionalanästhesie Erwähnung finden. Um eine Regionalanästhesie suffizient durchführen $\mathrm{zu}$ können, ist eine adäquate Ausbildung erforderlich, welche sich in der Regel kosten- und zeitintensiv gestaltet [5]. Dauerhafte Nervenschädigungen müssen ebenso als potenzieller Nachteil angeführt werden. Obwohl das Risiko als gering einzustufen ist [26], können solche Nebenwirkungen auftreten, welche normalerweise nicht unerhebliche medizinische Folgekosten nach sich ziehen. Weiters ist natürlich keine medizinische Methode $\mathrm{zu}$ $100 \%$ erfolgreich, und so sind insuffiziente regionale Blockaden besonders kostenintensiv, weil nachfolgend eine Allgemeinnarkose durchzuführen ist, 


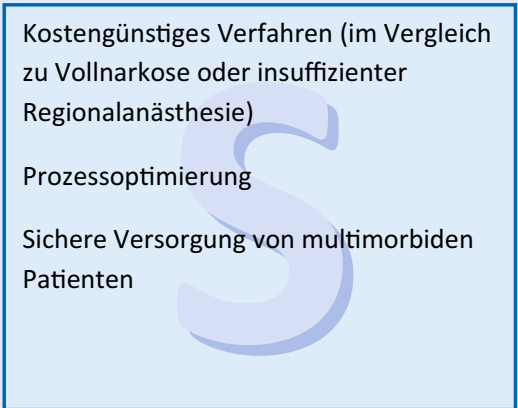

Steigerung des Kosten- und Effizienzbewusstseins im Gesundheitswesen

Zeitliche Optimierung der

Patientenversorgung

Trend zum ambulanten Operieren wird unterstützt

Rasche Operabilität von

Hochrisikopatienten

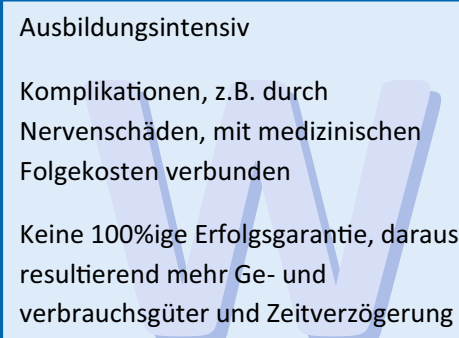

Ausbildungsintensiv

Komplikationen, z.B. durch

Nervenschäden, mit medizinischen

Folgekosten verbunden

Keine $100 \%$ ige Erfolgsgarantie, daraus

resultierend mehr Ge- und

verbrauchsgüter und Zeitverzögerung

Keine Bereitschaft des Personals für

Entwicklungs- und

Schulungsmaßnahmen

Interdisziplinäre Inakzeptanz

Fehlende Patientencompliance

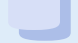

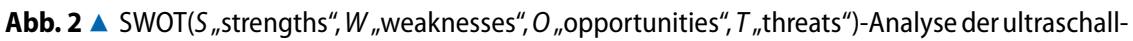
gezielten Regionalanästhesie aus ökonomischer Sicht

was zu signifikantem Zeitverlust und Mehrverbrauch an Personalressourcen, Ge- und Verbrauchsgütern sowie anästhesierelevanten Anlagegütern führt.

Die Analyse von Stärken und Schwächen führt zu den Chancen eines medizinischen Verfahrens. Das Erkennen von Chancen kann in Stärke umgewandelt werden, und die Notwendigkeit für bewusstes Handeln wird erkannt. Erst die Analyse vieler Einzelfaktoren lässt große Zusammenhänge erkennen. So ist die Planbarkeit elektiver (aber auch semiakuter) Operationen in Regionalanästhesie ein großer Vorteil. Zusätzlich können z. B. Nüchterngrenzen bei Operationen in reiner Regionalanästhesie großzügig betrachtet werden, wodurch Patienten rascher der operativen Versorgung zugeführt werden können und generell weniger Schmerzen (während der Wartezeit) haben. Optimale Abläufe von Operationen in ausschließlicher Regionalanästhesie unterstützen im Übrigen den Trend zur ambulanten Chirurgie. Unerwartete stationäre Aufnahmen in der ambulanten Chirurgie, die z. B. durch „postoperative nausea and vomiting" (PONV) verursacht werden, können in Regionalanästhesie praktisch ausgeschlossen werden.

\section{Praktische Konsequenzen}

Die betrieblichen Strukturen in Krankenhäusern sind durch funktionsorientierte Sichtweisen geprägt. Einzelne Bereiche konzentrieren sich auf die Erbringung ihrer Leistungen, eine den gesamten Behandlungsprozess begleitende Sichtweise fehlt zumeist. Dementsprechend finden Prozessoptimierungen, wenn überhaupt, nur in Organisationseinheiten statt und enden spätestens beim Übergang zwischen den Bereichen. Kernprozesse in Krankenhäusern erfahren dabei kaum Unterstützung bei der Planung, Durchführung und Überwachung [21]. Dies betrifft auch den Wissenstransfer, der über Schulungen des Personals in die Abteilungen und damit in die tägliche klinische Praxis kommen soll. Dies bedeutet initial einen Aufwand (der in den oben dargestellten Berechnungen nicht berücksichtigt wurde), ist jedoch real eine Investition in Prozesse, die sich schlussendlich in Kostenreduktion widerspiegeln.

Neben den direkten ökonomischen Aspekten ergeben sich noch andere Vorteile von suffizient angewendeter Regionalanästhesie, begleitet von den notwendigen strukturellen Anpassungen. Ein zentraler Aspekt ist der in den Fallvignetten deutlich erkennbare Zeitvorteil der Regionalanästhesie. Die rein anästhesiologisch-kontrollierte Zeitersparnis im Operationssaal betrug $15 \mathrm{~min}$ (๑ Tab. 1: suffiziente Regionalanästhesie vs. primäre Allgemeinnarkose; Einleitung der Allgemeinnarkose (5 $\mathrm{min})+$ Ausleitung der Allgemeinnarkose (8 min) + Setzen der Venenverweilkanüle $(2 \mathrm{~min}))$. Dies bedeutet bei 4 Fällen $1 \mathrm{~h}$ Zeitgewinn. Somit könnten die teilweise sehr langen OP-Wartezeiten (z. B. in der Orthopädie) [6] signifikant reduziert werden, was wiederum positive ökonomische Effekte in der präoperativen Betreuung dieser Patienten (z. B. Schmerztherapie, psychische Belastungsreaktionen etc.) mit sich bringen würde.

Die praktische Implementierung kosteneffizienter anästhesiologischer Verfahren ist mit Herausforderungen verbunden und wird im Sinne des Change management [14] anfangs auf Ablehnung vieler Mitarbeiter stoßen. Transparenz, Mitarbeiterförderung und Personalent- 
wicklung sind daher in der Etablierung von Standardverfahren durch Neubewertungen von wichtigen Aspekten (wie z. B. die Berücksichtigung aller relevanten Gesichtspunkte finanzieller Implikationen von medizinischen Verfahren) essenziell.

\section{Fazit für die Praxis}

- Die Bedeutung der ultraschallgezielten Regionalanästhesie im Kontext der perioperativen Versorgung von PatientInnen ist aus heutiger Sicht unbestritten.

- Ökonomischen Aspekten suffizienter Regionalanästhesie wurden bisher zu wenig Aufmerksamkeit geschenkt.

- Suffiziente Regionalanästhesie erscheint im Vergleich zur Allgemeinanästhesie als ökonomisch günstig. Am ungünstigsten erweist sich die insuffiziente Regionalanästhesie, die durch ein allgemeinanästhesiologisches Verfahren supplementiert werden muss.

- Voraussetzungen für suffizient durchgeführte regionalanästhesiologische Techniken sind strukturelle Adaptationen, welche in erster Linie die optimierte Ausbildung und Analysen des spezifischen Prozessmanagements erfordern.

\section{Korrespondenzadresse

Prof. Dr. P. Marhofer
Klinische Abteilung für
Anästhesie, allgemeine
Intensivmedizin und
Schmerztherapie,
Medizinische Universität
Wien
Spitalgasse 23, 1090 Wien,
Österreich
peter.marhofer@
meduniwien.ac.at

Funding. Open access funding provided by Medical University of Vienna.

\section{Einhaltung ethischer Richtlinien}

Interessenkonflikt. P. Marhoferist Mitglied des Editorial Boards des British Journal of Anaesthesia. M. Kraus und D. Marhofer geben an, dass kein Interessenkonflikt besteht.
Für diesen Beitrag wurden von den Autoren keine Studien an Menschen oder Tieren durchgeführt. Für die aufgeführten Studien gelten die jeweils dort angegebenen ethischen Richtlinien.

Open Access Dieser Artikel wird unter der Creative Commons Namensnennung 4.0 International Lizenz (http://creativecommons.org/licenses/by/4.0/deed. de) veröffentlicht, welche die Nutzung, Vervielfältigung, Bearbeitung, Verbreitung und Wiedergabe in jeglichem Medium und Format erlaubt, sofern Sie den/die ursprünglichen Autor(en) und die Quelle ordnungsgemäßnennen, einen Linkzur Creative Commons Lizenz beifügen und angeben, ob Änderungen vorgenommen wurden.

\section{Literatur}

1. Bachner F, Bobek J, Habimana Ket al (2018) Austria: health system review. Health Syst Transit 20:1-256

2. Basakha M, Yavari K, Sadeghi H et al (2014) Health care cost disease as a threat to Iranian aging society. J Res Health Sci 14:152-156

3. Brull R, Perlas A, Chan VW (2007) Ultrasoundguided peripheral nerve blockade. Curr Pain Headache Rep 11:25-32

4. Bundesministerium für Arbeit, Gesundheit und Konsumentenschutz, Bereich VIII/B (2018) Krankenanstalten in Zahlen. Überregionale Auswertung der Dokumentation der landesgesundheitsfondsfinanzierten Krankenanstalten. https://www.sozialministerium. at/cms/site/attachments/9/0/9/CH3984/ CMS1289376034962/oesterreich_2017.pdf. Zugegriffen: November 2018

5. Chen XX, Trivedi V, AISaflan AA et al (2017) Ultrasound-guided regional anesthesia simulation training: a systematic review. Reg Anesth Pain Med 42:741-750

6. Czypionka T, Kraus M, Röhrling G (2013) Wartezeiten auf Elektivoperationen - Neues zur Frage der Transparenz

7. Dirks U (2012) Aufgabenformate - Das Genre "Fallvignette"

8. Dresselhaus TR, Peabody JW, Lee $M$ et al (2000) Measuring compliance with preventive care guidelines: standardized patients, clinical vignettes, and the medical record. J Gen Intern Med 15:782-788

9. Fredrickson MJ, Krishnan S, Chen CY (2010) Postoperative analgesia for shoulder surgery: a critical appraisal and review of current techniques. Anaesthesia 65:608-624

10. Gonano C, Kettner SC, Ernstbrunner M et al (2009) Comparison of economical aspects of interscalene brachial plexus blockade and general anaesthesia for arthroscopic shoulder surgery. Br J Anaesth 103:428-433

11. Hartwig J, Kraemer H (2018) Baumolsche Kostenkrankheit im Schweizerischen Gesundheitswesen. Schweiz Ärzteztg 99:874-877

12. Kessler J, Marhofer P, Rapp HJ et al (2007) Ultrasound-guided anaesthesia of peripheral nerves. The new challenge for anaesthesiologists. Anaesthesist 56:642-655

13. Klucka J, Stourac P, Stouracova A et al (2017) Compartment syndrome and regional anaesthesia: critical review. Biomed Pap Med Fac Univ Palacky Olomouc Czech Repub 161:242-251

14. Lauer T (2014) Auslöser von Unternehmenswandel. In: Lauer T (Hrsg) Change Management,
Grundlagen und Erfolgsfaktoren. Springer-Gabler Aschaffenburg, S13-26

15. Marhofer P, Fritsch G (2017) Safe performance of peripheral regional anaesthesia: the significance of ultrasound guidance. Anaesthesia 72:431-434

16. Marhofer P, Harrop-Griffiths W, Kettner SC et al (2010) Fifteen years of ultrasound guidance in regional anaesthesia: part $1 . \mathrm{Br} J$ Anaesth 104:538-546

17. Marhofer $P$, Harrop-Griffiths $W$, Willschke $\mathrm{H}$ et al (2010) Fifteen years of ultrasound guidance in regional anaesthesia: part 2-recent developments in block techniques. Br J Anaesth 104:673-683

18. Marhofer P, Schrögendorfer K, Andel H et al (1998) Combined sciatic nerve 3-in-1 block in a high risk patient. Anasthesiol Intensivmed Notfallmed Schmerzther 33:399-401

19. OECD (2019) OECD Health Statistics. https://www. oecd.org/els/health-systems/health-data.htm. Zugegriffen: 2. Juli 2019

20. Peabody JW, Luck J, Glassman P et al (2000) Comparison of vignettes, standardized patients, and chart abstraction: a prospective validation study of 3 methods for measuring quality. JAMA 283:1715-1722

21. Reichert M (2000) Prozessmanagement im Krankenhaus: Nutzen, Anforderungen und Visionen. Krankenhaus 92:903-909

22. Schäfer MK, Wittenmeier E (2003) Ambulatory and day surgery. Anaesthesist 52:1046-1054

23. Statistik Austria (2019) Pressemitteilung: 11.958024/19. Laufende Gesundheitsausgaben liegen im Jahr 2017 unverändert bei 10,4\% des Bruttoinlandsprodukts. http://www.statistikaustria.at/web_de/statistiken/menschen_und gesellschaft/gesundheit/gesundheitsausgaben/ 120234.html.Zugegriffen: 12.2.2019

24. Teoli D, An J (2019) SWOT Analysis

25. van Geffen GJ, McCartney CJ, Gielen M et al (2007) Ultrasound as the only nerve localization technique for peripheral nerve block. J Clin Anesth 19:381-385

26. Wiesmann T, Döffert J, Steinfeldt T (2018) Peripheral regional anesthesia without any complications-a dream comes true?! Anasthesiol Intensivmed Notfallmed Schmerzther 53:252-268 